\title{
Gamma-Ray Bursts and its afterglow modeling
}

\section{Jessymol K Thomas*}

University of Johannesburg, Department of Physics, Auckland Park 2006, Johannesburg, South Africa

E-mail: jessymolkteuj.ac.za

\section{Reetanjali Moharana}

University of Johannesburg, Department of Physics, Auckland Park 2006, Johannesburg, South Africa

E-mail: reetanjalim@uj.ac.za

\section{Soebur Razzaque}

University of Johannesburg, Department of Physics, Auckland Park 2006, Johannesburg, South Africa

E-mail: srazzaque@uj.ac.za

Gamma Ray Bursts (GRBs) are the explosions which take place in the universe with the emission of extraordinarily high energies of the order of $10^{55} \mathrm{erg}$. These explosions were detected at redshift up to $\mathrm{z}=9.4$. Many theoretical models were used to explain the mechanism of GRBs. Here we have done the synchrotron modeling of the spectral energy distribution of the GRBs in the fast cooling and slow cooling regimes for the adiabatic blastwaves in Interstellar Medium (ISM) and wind environment. We have modeled the spectral energy distribution at different time intervals for GRB 130427A. GRBs help us to learn about the processes that cannot be studied under laboratory conditions and also enable us to understand the physics behind the formation and structure of matter in the Universe. GRBs are candidate sources of ultra high energy cosmic rays (UHECRs). High energy neutrinos can be produced by the interaction of these UHECRs with the GRB afterglow photons, which are produced in the blastwave.

SALT Science Conference 2015 -SSC2015-

1-5 June, 2015

Stellenbosch Institute of Advanced Study, South Africa

\footnotetext{
* Speaker.
} 


\section{Introduction}

The study of Gamma Ray Bursts (GRBs) are one of the most interesting fields in modern Astronomy and Astrophysics. GRBs are the explosions with gamma rays of energy $\sim 10^{55}$ ergs. Generally they have a duration of few seconds to hours. In GRBs the prompt emissions are accompanied by long lasting afterglows at all electromagnetic (EM) wavelengths. These afterglow photons are believed to be the synchrotron radiation from accelerated electrons in the blastwave shocks. Here we have done the synchrotron modeling of Spectral Energy Distributions (SEDs) for GRBs in the fast cooling regime and slow cooling regime in both Interstellar Medium (ISM) and wind environment. We have applied this modeling to one of the nearest and most energetic GRB detected on 27th of April 2013 with redshift $\mathrm{z}=0.34$ [1].

\section{Synchrotron modeling of GRBs}

GRBs are reasonably described by the synchrotron modeling and these models are based on the synchrotron emission of the relativistic, accelerated electrons in the magnetic field of the GRBs $[2,3]$. The accelerated electrons which cause the prompt emissions are produced by the internal shocks. Afterglows are produced when the external shocks from the blast waves collide with ISM and wind environment.

The typical energy of synchrotron photon as well as the synchroton cooling time of electrons depend on the lorentz factor $\gamma_{e}$ of the relativistic electron. To get the overall instantaneous synchrotron spectrum of electrons we need to model the blast wave evolution. $\gamma_{e, \min }$ and $\gamma_{e, c}$ are the minimum lorentz factor and the cooling lorentz factor of electrons producing synchrotron frequencies $v_{m}$ and $v_{c}$, respectively. The synchrotron self absorption frequency can be written as $v_{a}$.

The instantaneous synchrotron spectrum for a power law distribution of electrons is expressed in terms of the overall observed flux $F_{v}$ [2].

(a) Fast cooling $\left(\gamma_{e, c}<\gamma_{e, m i n}\right)$ : In the fast cooling regime the typical electron is cooling rapidly so $v_{c}<v_{m}$. The instantaneous synchrotron spectrum in this regime is given as:

$$
F_{v}=F_{v, \text { max }} \begin{cases}\left(\frac{v_{a}}{v_{c}}\right)^{\frac{1}{3}}\left(\frac{v}{v_{a}}\right)^{2} ; & v<v_{a}, \\ \left(\frac{v}{v_{c}}\right) \frac{1}{3} ; & v_{a}<v<v_{c}, \\ \left(\frac{v}{v_{c}}\right)^{\frac{-1}{2}} ; & v_{c}<v<v_{m}, \\ \left(\frac{v_{m}}{v_{c}}\right)^{\frac{-1}{2}}\left(\frac{v}{v_{m}}\right)^{\frac{-p}{2}} ; & v_{m}<v\end{cases}
$$

(b) Slow cooling $\left(\gamma_{e, c}>\gamma_{e, \min }\right)$ or $v_{c}>v_{m}$ : In case of slow cooling only the high energy tail of the distribution cools effectively and the synchrotron spectrum in this regime is given as:

$$
F_{v}=F_{v, \text { max }} \begin{cases}\left(\frac{v}{v_{a}}\right)^{2}\left(\frac{v_{a}}{v_{m}}\right)^{\frac{1}{3}} ; & v<v_{a}, \\ \left(\frac{v}{v_{m}}\right)^{\frac{1}{3}} ; & v_{a}<v<v_{m}, \\ \left(\frac{v}{v_{m}}\right)^{\frac{-(p-1)}{2}} ; & v_{m}<v<v_{c}, \\ \left(\frac{v_{c}}{v_{m}}\right)^{\frac{-(p-1)}{2}}\left(\frac{v}{v_{c}}\right)^{\frac{-p}{2}} ; & v_{c}<v\end{cases}
$$

\subsection{Spectral Energy Distribution in the ISM and wind - slow cooling and fast cooling regime}

The synchrotron modeling of a GRB afterglow is done for constant density Inter Stellar Medium (ISM) and wind environment in the fast cooling and slow cooling regime. The differ- 
ent synchrotron frequencies in ISM are as follows [4, 5]:

$$
\begin{gathered}
v_{m}=1.644 \times 10^{14} \varepsilon_{b, 0.1}^{1 / 2} \varepsilon_{e, 0.1}^{2} E_{55}^{1 / 2}(1+z)^{1 / 2} t_{d}^{-3 / 2} \mathrm{~Hz}, \\
v_{c}=1.931 \times 10^{13} \varepsilon_{b, 0.1}^{-3 / 2} E_{55}^{-1 / 2}(1+z)^{-1 / 2} t_{d}^{-1 / 2} n_{0}^{-1} \mathrm{~Hz}, \\
v_{a}=5.53 \times 10^{9} \varepsilon_{b, 0.1}^{1 / 5} \varepsilon_{e, 0.1}^{-1} E_{55}^{1 / 5}(1+z)^{-1} n_{0}^{3 / 5} \mathrm{~Hz} \\
v_{s}=5.334 \times 10^{22} n_{0}^{-1 / 8} E_{55}^{1 / 8}(1+z)^{-5 / 8} t_{d}^{-3 / 8} \phi_{1}^{-1} \mathrm{~Hz},
\end{gathered}
$$

and $F_{v, \max }$, the observed peak flux is given as:

$$
F_{V, \max }=7.95 \times 10^{-23}(1+z)^{-1} n_{0}^{1 / 2} d_{l, 28}^{-2} E_{55} \varepsilon_{b}^{1 / 2} \mathrm{erg} / \mathrm{cm}^{2} / \mathrm{s} / \mathrm{Hz},
$$

where, $n_{0}=1 / \mathrm{cm}^{3}$ is the particle density in the ISM $E_{55}$ is the initial kinetic energy of blast wave in $10^{55} \mathrm{erg}, \varepsilon_{e}$ is the electron equipartition fraction or the fraction of energy that goes to the relativistic electrons, $\varepsilon_{e, 0.1}=\varepsilon_{e} / 0.1, \varepsilon_{b}$ is the fraction of energy that goes to the magnetic energy $\varepsilon_{b, 0.1}=\varepsilon_{b} / 0.1, d_{l, 28}$ is the luminosity distance in $10^{28} \mathrm{~cm}$ unit and $t_{d}$ is the time after prompt emission in days. Equations derived for all the synchrotron frequencies in wind environment are given as in $[4,5]$ :

$$
\begin{gathered}
v_{m}=9.5 \times 10^{13} \varepsilon_{b, 0.1}^{1 / 2} \varepsilon_{e, 0.1}^{2} E_{55}^{1 / 2}(1+z)^{1 / 2} t_{d}^{-3 / 2} \mathrm{~Hz} \\
v_{c}=2.1 \times 10^{15} \varepsilon_{b, 0.1}^{-3 / 2} E_{55}^{1 / 2}(1+z)^{-3 / 2} t_{d}^{1 / 2} A_{*}^{-2} \mathrm{~Hz} \\
v_{a}=8.3 \times 10^{9} \varepsilon_{b, 0.1}^{1 / 5} \varepsilon_{e, 0.1}^{-1} E_{55}^{-2 / 5}(1+z)^{-2 / 5} t_{d}^{-3 / 5} A_{*}^{6 / 5} \mathrm{~Hz}, \\
v_{s}=8.22 \times 10^{22} A_{*}^{-1 / 4} E_{55}^{1 / 4}(1+z)^{-3 / 4} t_{d}^{-1 / 4} \phi_{1}^{-1} \mathrm{~Hz}, \\
F_{v, \text { max }}=3.53 \times 10^{-24} \varepsilon_{b, 0.1}^{1 / 2} E_{55}^{1 / 2}(1+z)^{-1 / 2} t_{d}^{-1 / 2} d_{l, 28}^{-2} \mathrm{erg} / \mathrm{cm}^{2} / \mathrm{s} / \mathrm{Hz},
\end{gathered}
$$

where, $A_{*} \equiv \dot{M}_{-5} / v_{8}$ corresponding to a mass-loss rate of $\dot{M}_{w}=10^{-5} \dot{M}_{-5} M_{\odot} y r^{-1}$ in wind by the progenitor star, with velocity $v_{w}=10^{8} v_{8} \mathrm{~cm} / \mathrm{s}$. The slow cooling and fast cooling can also be discriminated using transition time $T_{0} . T_{0}$ is the time at which the synchrotron frequencies $v_{m}$ become equal to the electron cooling frequency $v_{c}$. So above $T_{0}$ we get the slow cooling spectrum and below $T_{0}$ we get the fast cooling spectrum. We have calculated $T_{0}$ as $1.1 \times 10^{2} \mathrm{~s}$ for ISM and $3 \times 10^{2} s$ for wind media as shown in the frequency versus time plots in Fig 1 and Fig 2, respectively. Fig 3 and Fig 4 are the spectrum for the ISM and wind, respectively for different time intervals.

\section{The SED of GRB 130427A - wind model and ISM}

The GRB 130427A has got a highly energetic prompt emission and extremely bright afterglow in the range of X-rays, optical and Infra-red wavebands [6]. The optically thin slow cooling synchrotron spectra has been modeled for the GRB 130427A in the ISM and wind environment. The model is fitted with breaks at $v_{m}, v_{c}$ and $v_{s}$, the peak frequency, the cooling frequency and the 


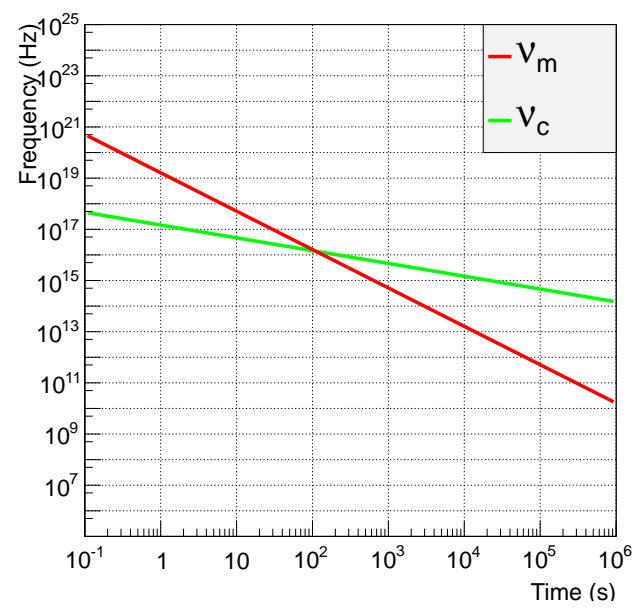

Figure 1: $T_{0}$ plot for ISM

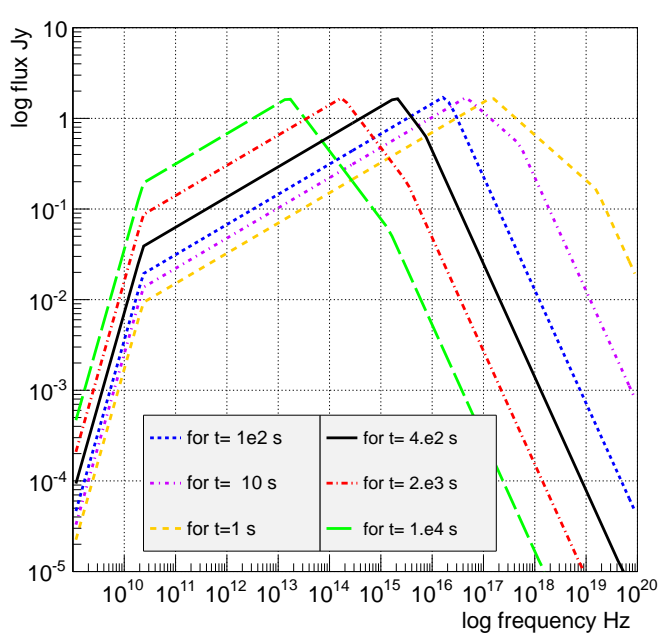

Figure 3: SED in ISM for fast cooling (times as in left box ) and slow cooling (times as in right box )

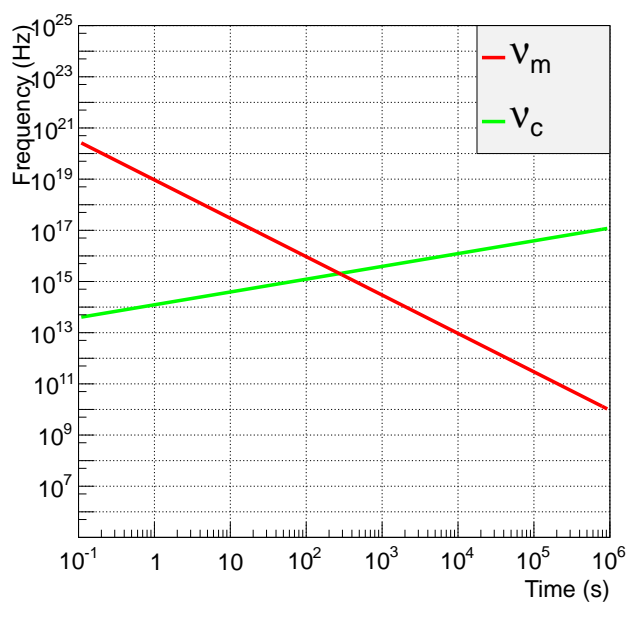

Figure 2: $T_{0}$ plot for WIND

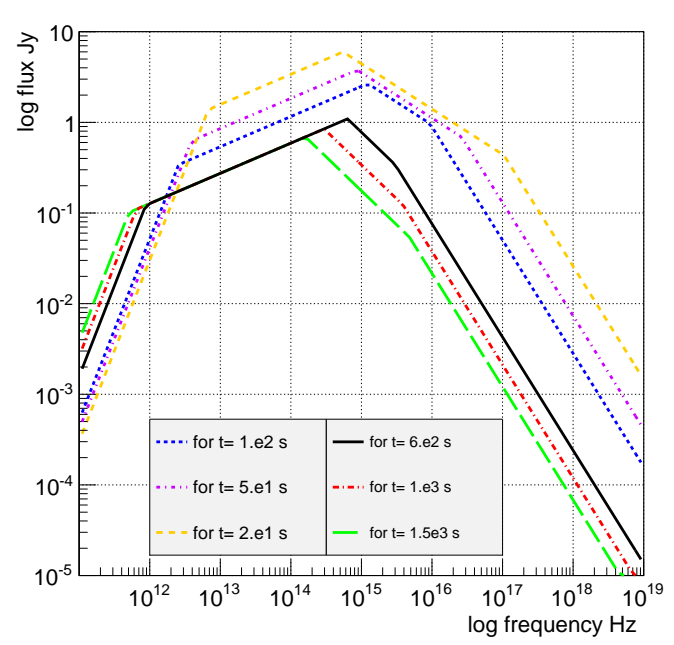

Figure 4: SED in WIND for fast cooling (times as in left box ) and slow cooling (times as in right box )

frequency corresponds to the highest energy, respectively.

The SED model for GRB 130427A for the slow cooling regime in the wind and the ISM media are plotted in Fig 5 and Fig 6, respectively. In Fig 5 the data observed by LAT, XRT-BAT and UVOT/optical for the GRB are plotted at different time intervals $193 \mathrm{~s}, 505 \mathrm{~s}, 4500 \mathrm{~s}$ and $23000 \mathrm{~s}$ and then fitted with synchrotron emission model for adiabatic blast waves in the wind environment. The model parameters we obtained are, $\varepsilon_{b}=0.036, \varepsilon_{e}=0.011, E_{55}=0.9, p=2.0, A *=0.01$, $z=0.34$ and $d_{L, 28}=d_{L} / 10^{28}=0.56$. Similarly for the ISM we fitted the data points with our model in Fig 6. The model parameters we obtained are $\varepsilon_{b}=1 \times 10^{-4}, \varepsilon_{e}=0.028, E_{55}=0.6$, $z=0.34$ and $d_{l, 28}=0.56, p=2.2$ and $n=0.01$. Our fitting result showed a good agreement with data in case of wind medium, however the spectrum did not fit well in case of ISM. 


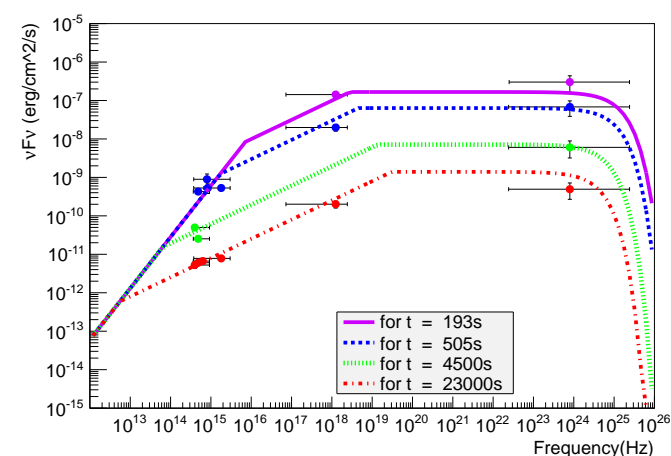

Figure 5: Spectral Energy Distribution in WIND medium is plotted for different time scales.

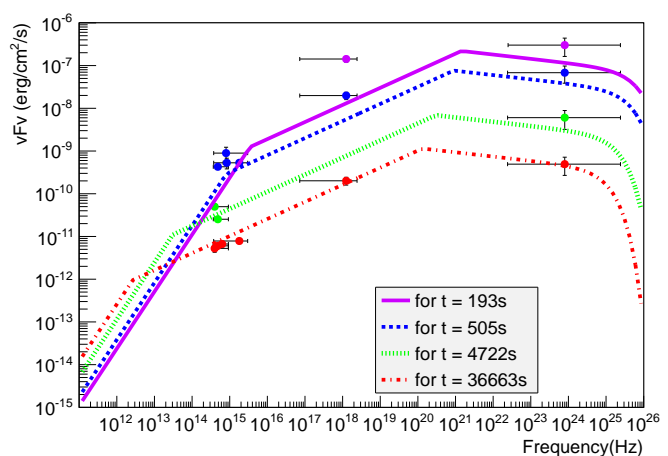

Figure 6: Spectral Energy Distribution in ISM is plotted for different time scales.

\section{Summary and outlook}

GRBs are extra-ordinarily energetic explosions and its modeling of the Spectral Energy Distributions can help us to explore them in detail. Synchrotron radiation of relativistic electrons is the important radiation mechanism in GRBs. We have done the synchrotron modeling of GRBs in the fast cooling and slow cooling regimes for the wind as well as ISM media. Synchrotron modeling of Spectral Energy Distribution for different time intervals were done for GRB 130427A in both ISM and wind media and is well desribed by wind environment. Through this modeling we have obtained reasonable parameters for GRB 130427A. GRBs are expected to be the sources of Ultra High Energy Cosmic Rays (UHECRs). Interaction of these UHECRs with afterglow photons could produce neutrinos. The model parameters would help us to calculate the neutrino flux from the GRBs and neutrinos can reveal the high energy processes in the explosions.

\section{References}

[1] Ackermann M et al. 2013 Fermi-LAT observations of GRB 130427A, Science 34342

[2] Piran T 2005 The Physics of Gamma-Ray Burst, Rev. Mod. Phys. 761143

[3] Meszaros p and Rees M J 1997 Optical and Long-Wavelength Afterglow from Gamma-Ray Bursts, Astrophys.J. 47623

[4] Razzaque S 2013, Long-lived PeV-EeV Neutrinos from GRB Blastwave, Phys. Rev. D 88103003

[5] Granot J, Piran T, Sari R 2010 Synchrotron self-absorption in Gamma-Ray Burst Afterglow, Astrophys.J. 527236

[6] A.Maselli et al 2013 GRB 130427A : A Nearby Ordinary Monster, science express 21 